\title{
Design and Simulation of Near-Terahertz GaN Photoconductive Switches--Operation in the Negative Differential Mobility Regime and Pulse Compression
}

This paper was downloaded from TechRxiv (https://www.techrxiv.org).

\section{LICENSE}

CC BY 4.0

SUBMISSION DATE / POSTED DATE

$15-01-2021 / 23-01-2021$

\section{CITATION}

Rakheja, Shaloo; Li, Kexin; Dowling, Karen M.; Conway, Adam; Voss, Lars (2021): Design and Simulation of Near-Terahertz GaN Photoconductive Switches--Operation in the Negative Differential Mobility Regime and Pulse Compression. TechRxiv. Preprint. https://doi.org/10.36227/techrxiv.13584953.v1

$\mathrm{DOI}$ 


\title{
Design and Simulation of Near-Terahertz GaN Photoconductive Switches-Operation in the Negative Differential Mobility Regime and Pulse Compression
}

\author{
Shaloo Rakheja, Member, IEEE, Kexin Li, Student Member, IEEE, Karen M. Dowling, Adam M. Conway \\ Member, IEEE, and Lars F. Voss
}

\begin{abstract}
The wide bandgap material, Gallium Nitride (GaN), has emerged as the dominant semiconductor material to implement high-electron mobility transistors (HEMTs) that form the basis of RF electronics. GaN is also an excellent material to realize photoconductive switches (PCSS) whose high-frequency performance could exceed that of RF HEMTs. In this paper, we numerically model the output characteristics of a GaN PCSS as a function of the input electrical and optical bias and the device dimensions. Importantly, we show that operating the GaN PCSS in the regime of negative differential mobility significantly benefits its high-frequency performance by compressing the temporal width of the output current pulse, while also enhancing its peak value. We find that when the optically excited carriers are generated in the middle of the active region, the bandwidth of the device is approximately 600 GHz, while delivering an output power exceeding $800 \mathrm{~mW}$ with a power gain greater than $35 \mathrm{~dB}$. The output power increases to $1.5 \mathrm{~W}$, and the power gain exceeds $40 \mathrm{~dB}$ with a near-terahertz bandwidth ( $\approx 800 \mathrm{GHz}$ ), as the laser source is moved closer to the anode. Finally, we elucidate that under high optical bias with significant electrostatic screening effects, the DC electric field across the device can be boosted to further enhance the performance of the GaN PCSS.
\end{abstract}

Index Terms-Optical triggering, Near-terahertz electronics, High-field transport, Negative differential mobility, Pulse compression, Wide bandgap semiconductors

\section{INTRODUCTION}

To meet the bandwidth, power, and gain demands of highspeed wireless communication systems [1], [2], new semiconductor technologies that can operate at frequencies exceeding $300 \mathrm{GHz}$, while delivering output power on the order of a Watt within a compact form-factor are highly sought after [3]. High electron mobility transistors (HEMTs) made from InP are capable of reaching frequencies higher than $300 \mathrm{GHz}$ but

This work was performed under the auspices of the U.S. Department of Energy by Lawrence Livermore National Laboratory under Contract DE-AC52-07NA27344, LLNL-JRNL-818156 and was supported by the LLNL-LDRD Program under Project No. 19-DR-015.

S. Rakheja and K. Li are with the Holonyak Micro and Nanotechnology Laboratory, Urbana, IL 61801 USA (e-mail: rakheja@illinois.edu).

K. M. Dowling, A. M. Conway, and L. F. Voss are with the Lawrence Livermore National Laboratory (e-mail: dowling7@IInl.gov). are limited to less than $10 \mathrm{~mW}$ of output power [4], [5]. Other technologies, such as Gunn diodes [6], [7], quantum cascade lasers [8], [9], plasmonic nano-antennas [10], [11], and backwards wave oscillators [12], have fundamental limits such as large device volume and/or low output power.

In this paper, we use Gallium Nitride $(\mathrm{GaN})$ to realize a new type of optically triggered photoconductive semiconductor switch (PCSS) that acts as both a high frequency generator and an amplifier while achieving peak power on the order of $1 \mathrm{~W}$. Our study focuses on the modeling of the transport of optically excited charge cloud in a GaN PCSS for a wide range of optical bias and device geometry parameters. Compared to conventional electronic devices, a PCSS is capable of generating extremely fast high-voltage pulses when exposed to electromagnetic radiation [13]. Encoding data into the signal generated by the PCSS is accomplished in a number of ways, for example, by dynamically varying the applied electric field, the optical path length of the laser, and/or the intensity of the laser, which impact both the frequency and amplitude of the output pulse. As a result of its extremely high breakdown electric field, ability to withstand harsh environments, and superior carrier transport properties (i.e., high electron mobility and saturation velocity), $\mathrm{GaN}$ is an excellent semiconductor material to implement a PCSS [14]. A unique attribute of $\mathrm{GaN}$ is that it exhibits negative differential mobility, wherein the drift velocity of free electrons decreases with increase in the DC electric field [15]. While the NDM effect in GaN is wellknown in the literature [16]-[18], this is the first work that utilizes the NDM regime to push the power-frequency bounds of GaN PCSS toward the sub-terahertz $(\mathrm{THz})$ regime (i.e., the frequency range of $500 \mathrm{GHz}$ to $1000 \mathrm{GHz}$ ).

Our analysis conclusively shows that we can simultaneously achieve pulse compression (for higher bandwidth) and increase in the peak output current (for higher output power and power gain) when the DC electric field across the GaN PCSS leads to NDM. The pulse compression is achieved due to the combined effect of NDM and field screening within the optically excited charge cloud, where the trailing electrons experience a lower electric field than the leading electrons. Through comprehensive simulations, we find that the $\mathrm{GaN}$ PCSS can deliver an output power of $\approx 830 \mathrm{~mW}$ with a power 
gain of $>35 \mathrm{~dB}$ while operating at a frequency of $\approx 600 \mathrm{GHz}$. These metrics are attained for a device with $100 \mu \mathrm{m}$ separation between the contacts, DC electric field of $250 \mathrm{kV} / \mathrm{cm}$, and when the device is illuminated at an equal distance from the electrodes with a laser of pulse width 10 ps, spot size 100 $\mathrm{nm}$, and generation rate $10^{28} \mathrm{~cm}^{-3} \mathrm{~s}^{-1}$. As the laser source is moved to a distance $12.5 \mu \mathrm{m}$ from the anode, the output power of the $100-\mu \mathrm{m} \mathrm{GaN} \mathrm{PCSS}$ increases to $\approx 1.5 \mathrm{~W}$, its power gain improves to $>40 \mathrm{~dB}$, while it continues to operate with near$\mathrm{THz}$ bandwidth $(\approx 800 \mathrm{GHz})$. The results presented here can guide experimental investigations of near-THz GaN PCSS in which the electric field and optical bias can be simultaneously engineered to benefit from the intrinsic NDM phenomenon.

The remainder of the paper is organized as follows. Section II focuses on the setup of the device and the modeling and simulation framework. The results of the carrier dynamics and device performance are discussed in Sec. III. The paper concludes in Sec. IV with a summary of key findings and prospects for future research and development.

\section{Device Setur and Modeling Framework}

The device setup of an optically excited GaN PCSS examined in this research is illustrated in Fig. 1(a). The incident laser is parameterized in terms of its generation rate, $G_{\mathrm{opt}}$, pulse width, $\Delta t$, and spot size, $S$. The power contained in the laser is given as $P_{\text {laser }}=$ $G_{\text {opt }} \mathcal{V}(h \nu) /\left[\eta_{Q}(1-r)\left(1-e^{-\alpha T}\right)\right]$, where $\mathcal{V}=W \times T \times S$ is the optically excited device volume ( $W$ and $T$ represent the width and the thickness, respectively, of the device), $h \nu$ is the photon energy, $r$ is the surface reflectance, $\alpha$ is the absorption coefficient, and $\eta_{Q}$ is the quantum efficiency. Here, we consider the PCSS is illuminated with a above-bandgap laser that has a wavelength of $365 \mathrm{~nm}$. The surface reflectance of $\mathrm{GaN}$ at $365 \mathrm{~nm}$ is 0.2 at $295 \mathrm{~K}$ [19]. Therefore, the laser power is $6.8 \mathrm{~mW}$ for a $1 \mu \mathrm{m}^{3}$ volume illumination and $G_{\text {opt }}=10^{28} \mathrm{~cm}^{-3} \mathrm{~s}^{-1}$. Here, we consider that $\alpha T \gg 1$. For all simulations presented here, the DC electric field $\mathcal{E}_{x} \in$ $[120,500] \mathrm{kV} / \mathrm{cm}, G_{\text {opt }} \in\left[10^{27}, 10^{29}\right] \mathrm{cm}^{-3} \mathrm{~s}^{-1}, \Delta t \in[1,10]$ ps, and $S=100 \mathrm{~nm}$. The quantum efficiency is assumed to be unity.

The DC electric field spans the NDM regime in which $d v_{n} / d \mathcal{E}_{x}<0$ ( $v_{n}$ is the drift velocity of electrons), which helps in controlling the shape of the charge cloud. That is, the NDM effects lead to an increase in peak electron current, while compressing the temporal width of the charge cloud as the charge cloud drifts toward the anode. Both these effects, qualitatively illustrated in Fig. 1(b), allow for much higher bandwidths and output power density to be achieved. Additionally, the electrostatic screening effects due to high optical power can be controlled by increasing the DC electric field and thus the device performance can be further improved.

In our analysis, we consider that the device length, $L$, is in the range of $10 \mu \mathrm{m}$ to $100 \mu \mathrm{m}$. The width of the device is fixed to $1 \mu \mathrm{m}$ and its thickness is $0.2 \mu \mathrm{m}$, and the nonuniform distribution of carrier density along the device width and its thickness is neglected. Hence, the results obtained here are representative of devices in which the electron cloud is

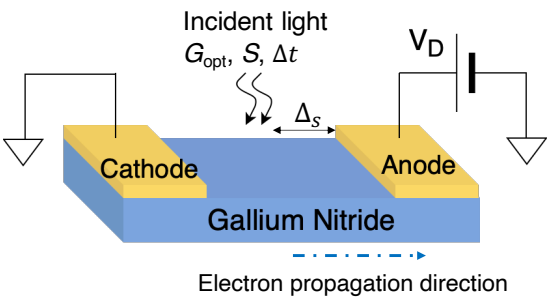

(a)

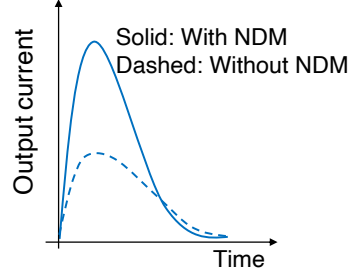

(b)
Fig. 1. (a) shows a lateral photoconductive switch using GaN as the active region. The incident light is parameterized using its generation rate, $G_{\text {opt }}$, spot size, $S$, and the pulse width $\Delta t$. The laser spot is located at a distance of $\boldsymbol{\Delta}_{s}$ from the anode. A bias of $V_{D}$ is applied across the device allowing electron current to be detected at the anode. (b) Due to the intrinsic negative differential mobility (NDM) effect in GaN, the pulse width of electron current can be compressed, while its peak value can be enhanced.

vertically confined such as in a heterostructure. Otherwise, the results presented here capture the upper bound of performance and can be used to examine the impact of input parameters (i.e., electrical and optical bias and device length) on the spatio-temporal profile of the charge cloud. Note that we focus mainly on the electron current since the NDM effect is absent for the valence band. However, a proper modeling of hole transport is essential as the electrons and holes are created in pairs via the above-bandgap laser. This implies that under the illumination region, electrons and holes will interact and recombine until they are extracted out of the contacts.

\section{A. Fundamental Modeling Equations}

The free carriers generated via optical excitation are transported toward opposite electrodes due to the applied bias, $V_{D}$. The detailed balance equation governing the transport of electrons and holes is given as [20]

$$
\begin{aligned}
& \nabla \cdot \overrightarrow{J_{n}}=-q \frac{\partial n}{\partial t}+q\left(R-G_{\mathrm{thm}, n}-G_{\mathrm{opt}, n}\right) \\
& \nabla \cdot \overrightarrow{J_{p}}=q \frac{\partial p}{\partial t}-q\left(R-G_{\mathrm{thm}, p}-G_{\mathrm{opt}, p}\right)
\end{aligned}
$$

where $\overrightarrow{J_{n}}\left(\overrightarrow{J_{p}}\right)$ is the electron (hole) current density, $n(p)$ is the electron (hole) density, $R$ is the carrier recombination rate, $G_{\mathrm{thm}, n}\left(G_{\mathrm{thm}, p}\right)$ is the thermal generation rate of electrons (holes), and $G_{\mathrm{opt}, n}\left(G_{\mathrm{opt}, p}\right)$ is the optical generation rate of electrons (holes). Since carriers are generated in pairs in the PCSS, the generation rate of electrons and holes is considered to be equal.

The particle current density is given as the sum of the drift and diffusion components:

$$
\begin{aligned}
& \overrightarrow{J_{n}}=\mu_{n}\left[n \nabla E_{c}\right]+D_{n}\left[\nabla n-n \nabla \ln \gamma_{n}\right] \\
& \overrightarrow{J_{p}}=\mu_{p}\left[p \nabla E_{v}\right]-D_{p}\left[\nabla p-p \nabla \ln \gamma_{p}\right]
\end{aligned}
$$

Here, $\mu_{n}\left(\mu_{p}\right)$ and $D_{n}\left(D_{p}\right)$ are the low-field mobility and diffusivity, respectively, of electrons (holes), $E_{c}$ is the bottom of the conduction band, $E_{v}$ is the top of the valence band, and $k_{B} T$ is the thermal energy. The parameters $\gamma_{n}$ and $\gamma_{p}$ account 
for the Fermi-Dirac statistics of carriers and are given as

$$
\begin{aligned}
\gamma_{n} & =\frac{n}{N_{c}} \exp \frac{E_{\mathrm{Fn}}-E_{c}}{k_{B} T}, \\
\gamma_{p} & =\frac{p}{N_{v}} \exp \frac{E_{\mathrm{Fp}}-E_{v}}{k_{B} T},
\end{aligned}
$$

where $N_{c}\left(N_{v}\right)$ is the effective conduction (valence) band density of states in $\mathrm{GaN}$, and $E_{\mathrm{Fn}}\left(E_{\mathrm{Fp}}\right)$ is the quasi-Fermi level of electrons (holes).

The recombination of carriers in $\mathrm{GaN}$ occurs via trapping centers and through band-to-band radiative recombination. The trap-assisted recombination is modeled using the ShockleyRead-Hall theory (SRH) and is given as [21], [22]

$$
R_{\mathrm{SRH}}=\frac{n p-\gamma_{n} \gamma_{p} n_{i}^{2}}{\tau_{h}\left(n+n_{1}\right)+\tau_{e}\left(p+p_{1}\right)},
$$

where $n_{i}$ is the intrinsic carrier density, $n_{1}=n_{i} e^{\frac{E_{\text {tr }}}{k_{B} T}}$ and $p_{1}=n_{i} e^{-\frac{E_{\mathrm{tr}}}{k_{B} T}}\left(E_{\mathrm{tr}}\right.$ is the energy level of the trap with respect to the intrinsic energy level of the semiconductor). The FermiDirac factors $\gamma_{n}$ and $\gamma_{p}$ are given in (3). Under the illumination region, the recombination rate is obtained by simplifying (4) under high-level injection (HLI) as $R \approx \Delta n / 2 \tau_{0}\left(\tau_{0}=\tau_{e}=\right.$ $\tau_{h}$ and $\Delta n \approx n$ is the excess carrier density). However, once the electrons and holes are separated due to the applied bias, the recombination rate becomes negligible as the background free carrier density is assumed to be negligible. The value of $\tau_{0}$ depends strongly on the threading dislocation density as a result of the heteroepitaxial growth of $\mathrm{GaN}$ on non-native substrates, as well as point defect (i.e., vacancy and impurity) concentration, which is subject to material growth chemistry and conditions. The non-radiative carrier lifetime in $\mathrm{GaN}$ on $\mathrm{Al}_{2} \mathrm{O}_{3}(\mathrm{Si})$ grown via metalorganic chemical vapor deposition (MOCVD) is reported to be $\approx 400 \mathrm{ps}(250 \mathrm{ps})$ at room temperature in [23]. The power-dependent photoluminescence measurements showed a strong yellow luminescence (YL) at $2.25 \mathrm{eV}$ in both $\mathrm{GaN}$ on $\mathrm{Al}_{2} \mathrm{O}_{3}$ and $\mathrm{GaN}$ on $\mathrm{Si}$ samples. The YL band was attributed to $\mathrm{V}_{\mathrm{Ga}}$ and $\mathrm{C}_{\mathrm{N}}$-related defects [23]. In [24], it was shown that for GaN epilayers deposited on high-quality AlN buffer grown on sapphire using migrationenhanced MOCVD, $\tau_{0} \approx 2 \mathrm{~ns}$ at room temperature. In homoepitaxial $\mathrm{GaN}$, the room temperature value of $\tau_{0}$ is reported to be $970 \mathrm{ps}$ [25], while a value of $860 \mathrm{ps}$ is reported in the GaN sample grown by lateral epitaxial growth [26]. For all results reported in this paper, $\tau_{0}=1 \mathrm{~ns}$ and $E_{\mathrm{tr}}=0$, unless otherwise noted.

The band-to-band radiative recombination rate is given as $R_{\text {rad }}=k_{\text {rad }}\left(n p-n_{i}^{2}\right)$, where $k_{\text {rad }}$ is the radiative band-toband recombination coefficient. The value of $k_{\text {rad }}$ is on the order of $10^{-11} \mathrm{~cm}^{-3} \mathrm{~s}^{-1}$ [27], [28]. Under the HLI condition, $R_{\mathrm{rad}} \approx k_{\mathrm{rad}} \Delta n^{2}$. For $\tau_{0}=1 \mathrm{~ns}$ and $k_{\mathrm{rad}}<10^{-10}$ $\mathrm{cm}^{3} \mathrm{~s}^{-1}$, the ratio of the trap-assisted recombination rate and the radiative recombination rate $\frac{R_{\mathrm{SRH}}}{R_{\mathrm{rad}}}=\frac{1}{2 \tau_{0} k_{\mathrm{rad}} \Delta n}>5$ even for the highest level of optical excitation used in our study: $G_{\mathrm{opt}}=10^{29} \mathrm{~cm}^{-3} \mathrm{~s}^{-1}$ and $\Delta t=10$ ps. Our analysis is corroborated in the result shown in Fig. 2, where we find that the trap-assisted recombination rate dominates at all times in the GaN PCSS unless $k_{\text {rad }}$ is chosen to be on the order of $10^{-9}$ $\mathrm{cm}^{3} \mathrm{~s}^{-1}$, which is approximately an order of magnitude higher

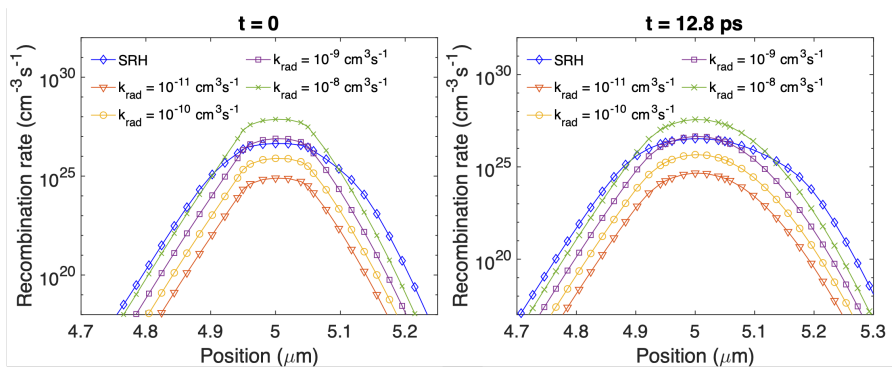

Fig. 2. Recombination rate due to $\mathrm{SRH}$ and radiative mechanisms at (a) $0 \mathrm{ps}$ and (b) $12.8 \mathrm{ps}$ in a $10-\mu \mathrm{m}$ long PCSS with $\boldsymbol{V}_{\boldsymbol{D}}=200 \mathrm{~V}, \boldsymbol{G}_{\mathrm{opt}}=$ $10^{29} \mathrm{~cm}^{-3} \mathrm{~s}^{-1}$ and $\Delta t=10 \mathrm{ps}$.

than the value of $k_{\text {rad }}$ reported in the literature. Since the trap-assisted recombination rate is significantly higher than the radiative recombination rate, we do not consider the radiative recombination further in our analysis.

The transport equations are solved self-consistently with Poisson's equation that governs the distribution of electric field lines due to the space charge $\rho$ according to

$$
\nabla \cdot(\epsilon \mathcal{E})=\rho=q\left(p-n+N_{D}^{+}-N_{A}^{-}\right)+\rho_{\text {trap }},
$$

where $\epsilon=\epsilon_{r} \epsilon_{0}$ is the semiconductor permittivity $\left(\epsilon_{0}=\right.$ $8.85 \times 10^{-12} \mathrm{~F} / \mathrm{m}$ and $\epsilon_{r}$ is the relative dielectric permittivity of the semiconductor), $N_{D}^{+}\left(N_{A}^{-}\right)$is the ionized donor (acceptor) concentration, and $\rho_{\text {trap }}$ is the charge density due to the traps and fixed charges. In this paper, we consider that the semiconductor is compensation doped to achieve a highly resistive state of the PCSS in the dark mode. The effect of $\rho_{\text {trap }}$ is considered only on the recombination kinetics but is otherwise excluded in our study. This allows us to determine the upper bound of performance for a GaN PCSS.

\section{B. High-Field Transport Characteristics}

At low electric fields $(\mathcal{E})$, the drift velocity of electrons $\left(v_{n}\right)$ increases linearly with $\mathcal{E}$. However, beyond a certain critical electric field, the electron velocity in $\mathrm{GaN}$ decreases with electric field, implying a negative value of the differential mobility (i.e., $d v_{n} / d \mathcal{E}<0$.) Using ensemble two-valley Monte-Carlo simulations, Ref. [29] showed that for an intervalley separation ranging from $1.2 \mathrm{eV}$ to $1.9 \mathrm{eV}$ in $\mathrm{GaN}$, the origin of the NDM effect is attributed to the scattering of electrons to a higher energy valley with a lower effective mass compared to the main valley. Hence, the NDM effect is also referred to as the transferred-electron effect (TEE). Reference [29] reports a peak electron drift velocity of $2.99 \times 10^{7} \mathrm{~cm} / \mathrm{s}$ at a critical electric field of $130 \mathrm{kV} / \mathrm{cm}$ in bulk GaN. In another theoretical work [30], the critical electric field for the onset of NDM effects in $\mathrm{GaN}$ was found to be $140 \mathrm{kV} / \mathrm{cm}$. Experimental data in [31] shows the NDM effect occurs at a critical electric field of $180 \mathrm{kV} / \mathrm{cm}$ with a peak electron drift velocity of $2.5 \times 10^{7}$ $\mathrm{cm} / \mathrm{s}$. In [16], a critical electric field of $191 \mathrm{kV} / \mathrm{cm}$ in $\mathrm{GaN}$ was experimentally observed using metal-semiconductor-metal structures. However, the NDM effect in [16] was only observed for bulk GaN samples with free carrier density $10^{14} \mathrm{~cm}^{-3}$, while NDM effect in semi-insulating GaN samples was not observed. 
To capture the NDM effect in GaN, we model the electron velocity dependence on the driving force $\left(\mathcal{F}_{\mathrm{hfs}, \mathrm{n}}\right)$ according to [32], [33]

$$
\begin{aligned}
v_{n} & =\mu_{n}\left(F_{\mathrm{hfs}, \mathrm{n}}\right) F_{\mathrm{hfs}, \mathrm{n}}, \\
\mu_{n}\left(F_{\mathrm{hfs}, \mathrm{n}}\right) & =\frac{\mu_{\mathrm{low}, \mathrm{n}}+v_{\mathrm{sat}, \mathrm{n}} \frac{F_{\mathrm{hfs}, \mathrm{n}}^{\beta-1}}{E_{1}^{\beta}}}{1+\gamma\left(\frac{F_{\mathrm{hfs}, \mathrm{n}}}{E_{0}}\right)^{\alpha}+\left(\frac{F_{\mathrm{hfs}, \mathrm{n}}}{E_{1}}\right)^{\beta}},
\end{aligned}
$$

where $\mu_{\text {low,n }}$ is the low-field electron mobility, $v_{\text {sat,n }}$ is the saturation velocity of electrons, $E_{0}$ refers to the electric field at which the drift velocity of electrons begins to saturate, $E_{1}$ $\left(<E_{0}\right)$ is the value of the electric field that corresponds to the onset of the NDM effect, and the parameters $\alpha, \beta$, and $\gamma$ are empirical in nature. Essentially, these empirical parameters control the rate of change of the drift velocity between the linear, NDM, and the saturation regimes. Per the model in (6), the peak drift velocity of electrons is given as $v_{n}^{\text {peak }}=$ $\left(\mu_{\text {low }, \mathrm{n}} E_{1}+v_{\text {sat }, \mathrm{n}}\right) /\left(2+\gamma\left(E_{1} / E_{0}\right)^{\alpha}\right)$.

The driving force for numerical simulations is considered to be the gradient of the quasi Fermi potential. That is,

$$
\begin{aligned}
F_{\mathrm{hfs}, \mathrm{n}} & =\left|\nabla \Phi_{n}\right|, \\
\Phi_{n} & =\phi-\phi_{\mathrm{ref}}+\frac{\chi}{q}-\phi_{t} \mathcal{F}_{-1 / 2}\left(\frac{n}{N_{c}}\right) .
\end{aligned}
$$

Here, $\phi$ is the electrostatic potential (i.e., the bending of the conduction and valence bands), $\phi_{\text {ref }}$ is chosen as the intrinsic level of the semiconductor, $\phi_{t}=k_{B} T / q$ is the thermal voltage, $\chi$ is the semiconductor electron affinity, $N_{c}$ is the effective conduction band density of states, and $\mathcal{F}_{-1 / 2}(x)$ is the FermiDirac integral of order $-1 / 2$.

Figure 3 shows the electron velocity versus electric field in $\mathrm{GaN}$ from various Monte-Carlo simulations. The fit of the model in (6) to the numerical data is also shown. The model parameters are: $\mu_{1}=1100 \mathrm{~cm}^{2} / \mathrm{Vs}, E_{0}=220 \mathrm{kV} / \mathrm{cm}, v_{\text {sat }}=$ $1.5 \times 10^{7} \mathrm{~cm} / \mathrm{s}, E_{1}=140 \mathrm{kV} / \mathrm{cm}, \gamma=6.2, \alpha=0.79$, and $\beta=7.2$

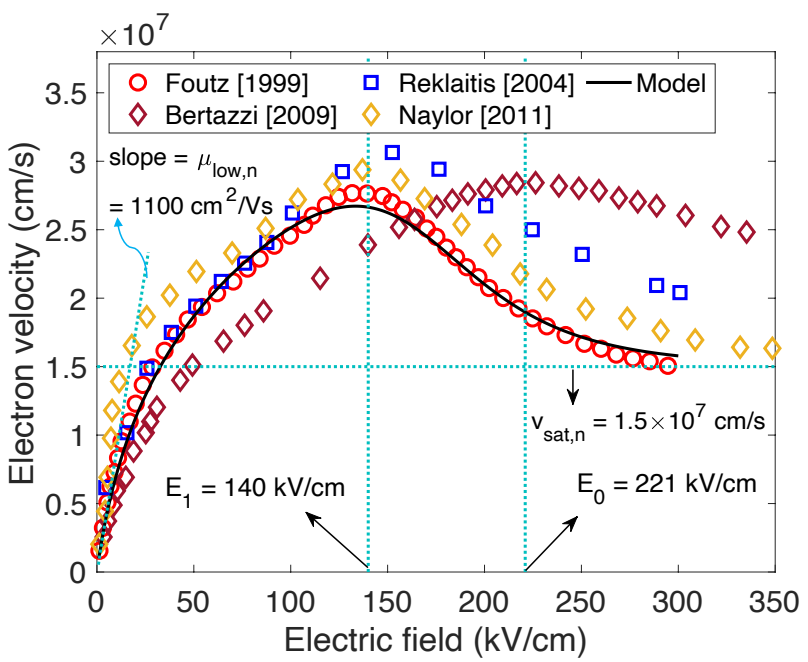

Fig. 3. Electron drift velocity versus electric field at room temperature. Symbols represent Monte-Carlo simulation data [29], [30], [34], [35], while the solid line is obtained using the model fit of (6).
Unlike the velocity of electrons, the velocity of holes in $\mathrm{GaN}$ shows complete saturation at a high driving force $\left(F_{\mathrm{hfs}, \mathrm{p}}\right)$ and is modeled as

$$
v_{p}=\frac{\mu_{\mathrm{low}, \mathrm{p}}}{\left[1+\left(\frac{\mu_{\mathrm{low}, \mathrm{p}} F_{\mathrm{hfs}, \mathrm{p}}}{v_{\mathrm{sat}, \mathrm{p}}}\right)^{\beta_{p}}\right]^{1 / \beta_{p}}},
$$

where $\mu_{\text {low,p }}$ is the low-field hole mobility, $v_{\mathrm{sat}, \mathrm{p}}$ is the hole saturation velocity, and $\beta_{p}$ is an empirical parameter that controls the sharpness of the transition of the velocity from the linear to the saturation regimes. Similar to the case of electrons, the driving force for electrons is considered to be the gradient of the hole quasi Fermi potential. According to the model in (8), the critical electric field for the velocity saturation of holes is $\mathcal{E}_{\text {crit }, \mathrm{p}}=v_{\text {sat }, \mathrm{p}} / \mu_{\text {low }, \mathrm{p}}$.

Figure 4 shows the hole velocity versus electric field obtained using Monte-Carlo simulations [36], [37] and experimental data [38] which uses photo-assisted method to determine hole velocity in a p-i-n diode. The model fit (Model1) to the data from [36] and [38] is fitted using (8) with $\mu_{\text {low }, \mathrm{p}}=17 \mathrm{~cm}^{2} / \mathrm{Vs}, v_{\text {sat }, \mathrm{p}}=6.63 \times 10^{6} \mathrm{~cm} / \mathrm{s}$, and $\beta_{p}=1.75$, while the model fit (Model-2) to the data from [37] uses $\mu_{\text {low }, \mathrm{p}}=60 \mathrm{~cm}^{2} / \mathrm{Vs}, v_{\mathrm{sat}, \mathrm{p}}=6.7 \times 10^{6} \mathrm{~cm} / \mathrm{s}$, and $\beta_{p}=1.2$. The critical field for velocity saturation of holes is $390 \mathrm{kV} / \mathrm{cm}$ for Model-1, while it is $112 \mathrm{kV} / \mathrm{cm}$ for Model-2.

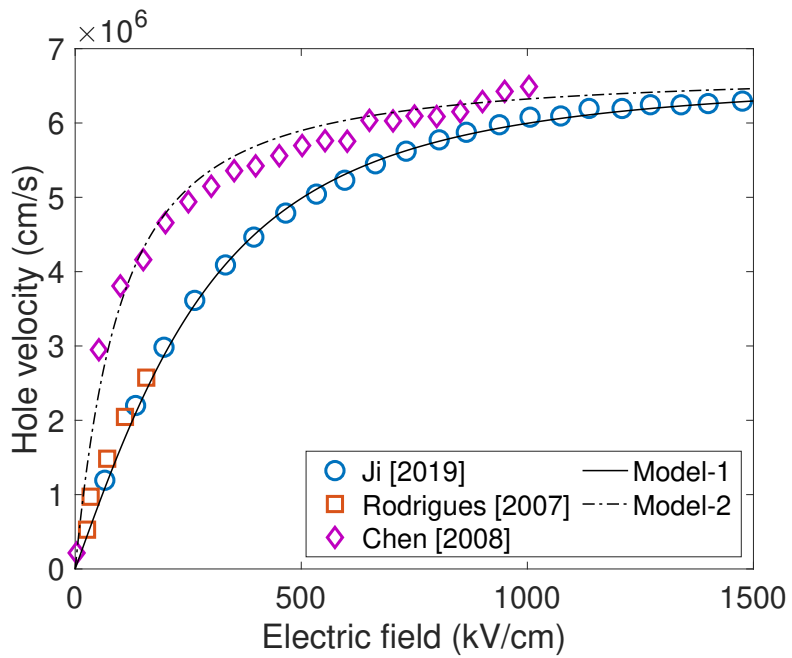

Fig. 4. Hole drift velocity versus electric field at room temperature. Monte-Carlo simulation data (squares and diamonds) are taken from [36], [37], while the experimental data (circles) is from [38]. The model fit is obtained using (8).

\section{NeAR-THz PERformance}

The electrical transport simulations are performed for the setup in Fig. 1(a) for a broad range of geometry and optical excitation parameters. We focus on the transport of electrons since the NDM effects are present only for the conduction band in GaN. Table I lists the parameters used for simulating the operation of GaN PCSS. For each set of input parameters, we obtain the following figure of merit (FoM) of the PCSS: (i) bandwidth $(\mathrm{BW})$, (ii) maximum output power $\left(P_{\text {out }, \mathrm{m}}\right)$, and (iii) maximum power gain, $G_{\mathrm{pm}}=P_{\mathrm{out}, \mathrm{m}} / P_{\text {laser }}$, where $P_{\text {laser }}$ 
TABLE I

MATERIAL PARAMETERS USED FOR SIMULATING THE OPERATION OF GAN PCSS. ALL MATERIAL PARAMETERS ARE REPORTED AT $300 \mathrm{~K}$. $m_{0}=\mathbf{9 . 1} \times 10^{-31} \mathrm{KG}$ IS THE FREE ELECTRON MASS.

\begin{tabular}{l|l}
\hline Parameter & Value \\
\hline Crystal structure & Wurtzite \\
Bandgap, $E_{g}$ & $3.4 \mathrm{e} \mathrm{V}$ \\
Energy separation between $\Gamma$ valley and M-L valleys & {$[1.1,1.9] \mathrm{eV}$} \\
Electron affinity, $\chi$ & $4.1 \mathrm{eV}$ \\
Static relative dielectric permittivity, $\epsilon_{r}$ & 8.9 \\
Effective conduction band density of states, $N_{c}$ & $2.8 \times 10^{18} \mathrm{~cm}^{-3}$ \\
Effective valence band density of states, $N_{v}$ & $4.6 \times 10^{19} \mathrm{~cm}^{-3}$ \\
Breakdown field, $\mathcal{E}_{\mathrm{br}}$ & $5 \times 10^{6} \mathrm{~V} / \mathrm{cm}$ \\
Low-field electron mobility, $\mu_{\text {low,n }}$ & $1100 \mathrm{~cm}^{2} / \mathrm{Vs}$ \\
Low-field hole mobility, $\mu_{\text {low,p }}$ & $17 \mathrm{~cm}^{2} / \mathrm{Vs}$ \\
Diffusivity of electrons, $D_{n}=\phi_{t} \mu_{\text {low,n }}$ & $28.3 \mathrm{~cm}^{2} / \mathrm{s}$ \\
Diffusivity of holes, $D_{p}=\phi_{t} \mu_{\text {low,p }}$ & $0.44 \mathrm{~cm}^{2} / \mathrm{s}$ \\
Thermal velocity of electrons, $v_{\text {th,n }}$ & $2.6 \times 10^{7} \mathrm{~cm} / \mathrm{s}$ \\
Thermal velocity of holes, $v_{\text {th,p }}$ & $9.4 \times 10^{6} \mathrm{~cm} / \mathrm{s}$ \\
Effective mass of electrons, $m_{\mathrm{eff}, \mathrm{n}}$ & $0.2 m_{0}$ \\
Effective mass of holes, $m_{\mathrm{eff}, \mathrm{p}}$ & $1.4 m_{0} \quad(\mathrm{heavy}) ;$ \\
& $0.3 m_{0} \quad(\mathrm{light})$, \\
& $0.6 m_{0}(\mathrm{split}-\mathrm{off})$ \\
Peak electron drift velocity & $2.6 \times 10^{7} \mathrm{~cm} / \mathrm{s}$ \\
Critical electric field for onset of NDM effect, $E_{1}$ & $140 \mathrm{kV} / \mathrm{cm}$ \\
Saturation drift velocity of holes, $v_{\mathrm{sat}, \mathrm{p}}$ & $6.63 \times 10^{6} \mathrm{~cm} / \mathrm{s}$ \\
Critical electric field for velocity saturation of holes, & $390 \mathrm{kV} / \mathrm{cm}$ \\
$E_{\mathrm{crit}, \mathrm{p}}=v_{\mathrm{sat}, \mathrm{p}} / \mu_{\text {low, }}$ & \\
Trap-assisted recombination lifetime, $\tau_{0}$ & $1 \mathrm{~ns}$ \\
\hline
\end{tabular}

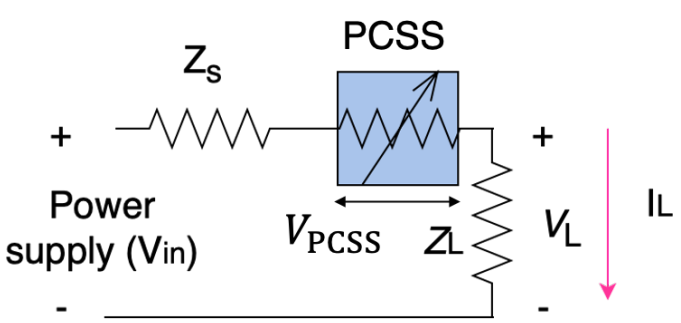

Fig. 5. Circuit model of GaN PCSS. $\boldsymbol{Z}_{\boldsymbol{s}}$ and $\boldsymbol{Z}_{\boldsymbol{L}}$ represent the source and load resistances, respectively. The voltage drop across the PCSS is $V_{\mathrm{PCSS}}$ and the current through the circuit is $\boldsymbol{I}_{\boldsymbol{L}}$. The input supply $V_{\mathrm{in}}$ is chosen such that for a given length of the PCSS, the average electric field is high enough to observe the NDM effect in GaN.

is the power contained in the incident laser. The $\mathrm{BW}$ of the PCSS is given as BW $=1 / 2 \mathrm{FWHM}$, where FWHM is the full width at half maximum of the electron current collected at the anode. To obtain $P_{\text {out,m }}$, we use the equivalent circuit of Fig. 5, where the PCSS is represented using a voltagecontrolled nonlinear resistor. The value of this resistor is given as $R_{\mathrm{PCSS}}=V_{\mathrm{PCSS}} / I_{L}$. Here, $V_{\mathrm{PCSS}}$ is the voltage across the resistor and $I_{L}=q W n v_{n}$ is the peak current through the circuit. Since both $n$ and $v_{n}$ depend on the PCSS voltage drop, the circuit model of Fig. 5 is justified.

According to the circuit model in Fig. 5, the power delivered to the load $Z_{L}$ is

$$
P_{L}=\frac{V_{L}^{2}}{Z_{L}}=V_{\mathrm{in}}^{2} \frac{Z_{L}}{\left(Z_{L}+Z_{s}+R_{\mathrm{PCSS}}\right)^{2}},
$$

where $V_{\text {in }}$ is the input power supply, $Z_{s}$ is the resistance of the power supply, and $V_{L}$ is the voltage across the load resistor. The output is maximized for $Z_{L} \approx R_{\mathrm{PCSS}}$ if $Z_{s}$ is negligible.
At the maximum power point, $V_{\mathrm{PCSS}}=V_{\mathrm{in}} / 2$ and

$$
\begin{aligned}
P_{\mathrm{out}, \mathrm{m}} & =\frac{V_{\mathrm{in}}^{2}}{4 R_{\mathrm{PCSS}}\left(V_{\mathrm{PCSS}}=V_{\mathrm{in}} / 2\right)} \\
& =\frac{V_{\mathrm{in}}}{2} I_{L}\left(\frac{V_{\mathrm{in}}}{2}\right)=V_{\mathrm{PCSS}} I_{L}\left(V_{\mathrm{PCSS}}\right) .
\end{aligned}
$$

Knowing the maximum output power and the laser power allows us to obtain the maximum power gain of the PCSS:

$$
G_{\mathrm{pm}}(\mathrm{dB})=10 \log _{10} \frac{P_{\mathrm{out}, \mathrm{m}}}{P_{\text {laser }}}=10 \log _{10} \frac{V_{\mathrm{PCSS}} I_{L}\left(V_{\mathrm{PCSS}}\right)}{G_{\mathrm{opt}} \mathcal{V} h \nu} .
$$

\section{A. Central location of the laser source}

In this section, we consider that the laser source generates carriers in the middle of the channel and there is no offset. That is, $\Delta_{s}=L / 2$ (see Fig. 1). The temporal evolution of the electron current density at the anode is shown in Fig. 6 for $G_{\mathrm{opt}}=10^{28} \mathrm{~cm}^{-3} \mathrm{~s}^{-1}, L=10 \mu \mathrm{m}$, and laser pulse width ranging from 1 ps to 10 ps. There exists a critical DC bias at which the output current is maximized, while the pulse width of the electron current is minimized. This critical bias corresponds to the onset of the NDM effect in GaN. Because of field screening within the cloud, the trailing electrons experience a lower electric field than the leading electrons. This negates the effect of diffusion and results in the trailing electrons traveling at higher velocity, compressing the size of the electron cloud. When the signal reaches the anode, the output current pulse is compressed, and the peak power is amplified compared to the initially generated cloud as shown in Fig. 7.

The value of the critical bias at which pulse compression is observed strongly depends on the pulse width and the optical generation rate of the laser. As $G_{\mathrm{opt}}$ and $\Delta t$ increase, the concentration of optically excited free carriers increases, which enhances the electrostatic screening effects. To counter the screening effects and observe NDM, larger DC electric fields are required, which underscores the observation of Fig. 6. That is, the DC bias at which the pulse compression and peak output current are observed for $G_{\mathrm{opt}}=10^{28} \mathrm{~cm}^{-3} \mathrm{~s}^{-1}$ increases from $170 \mathrm{~V}$ for $\Delta t=1$ ps to $250 \mathrm{~V}$ for $\Delta t=10 \mathrm{ps}$ for a $10-\mu \mathrm{m}$ long PCSS.

To further corroborate the non-monotonic dependence of the peak output current on the DC bias, we compare the optically excited electron density along the device length at various time instances $(t)$, where $t=0$ corresponds to the instant when the laser is turned off. As shown in Fig. 8, at $t=0$, the peak electron density follows that $n_{\text {peak }}(190 \mathrm{~V})>n_{\text {peak }}(170 \mathrm{~V})$. However, due to the NDM effect, the drift velocity of electrons scales as $v_{n}(190 \mathrm{~V})<v_{n}(170 \mathrm{~V})$. Hence, the arrival time of the peak of the charge cloud at the anode is higher for $190 \mathrm{~V}$ bias. Due to the slow propagation speed at $190 \mathrm{~V}$, the charge cloud suffers more from diffusion effects which leads to the observation that the peak output current is lower for $190 \mathrm{~V}$ than for $170 \mathrm{~V}$ bias (see Fig. 6(a)).

To explore the interplay between the NDM effect and electrostatic screening, in Fig. 9, we plot the electric field along the PCSS for various time points and several different cases of optical excitation. As the product $G_{\mathrm{opt}} \times \Delta t$ increases, 


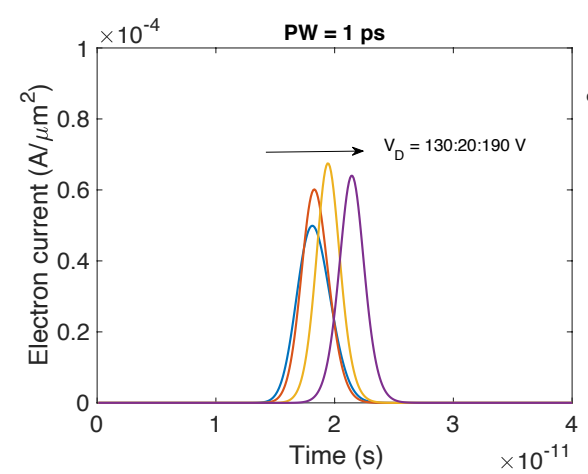

(a)

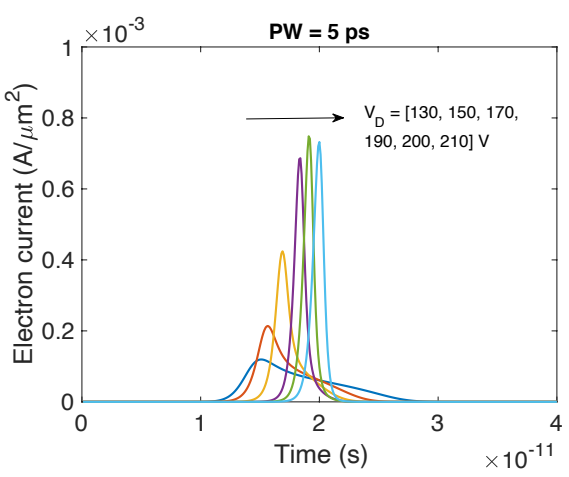

(b)

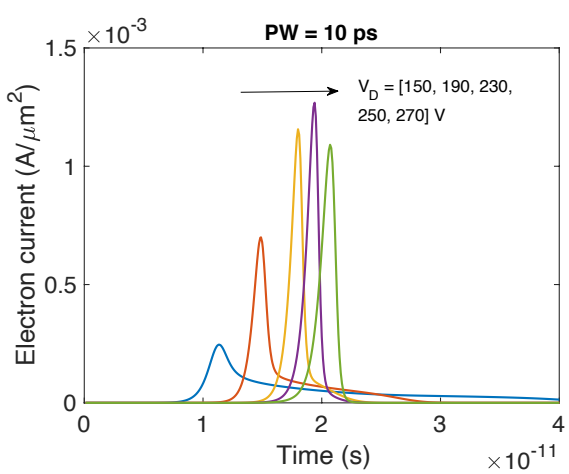

(c)

Fig. 6. Temporal evolution of the electron current at the anode for $G_{\mathbf{o p t}}=\mathbf{1 0}^{\mathbf{2 8}} \mathrm{cm}^{-\mathbf{3}} \mathrm{s}^{-1}, S=100 \mathrm{~nm}, \boldsymbol{L}=\mathbf{1 0} \boldsymbol{\mu m}$, and pulse width (PW) of 1 ps, 5 ps, 10 ps. $V_{\boldsymbol{D}}$ is the DC bias applied to the PCSS.

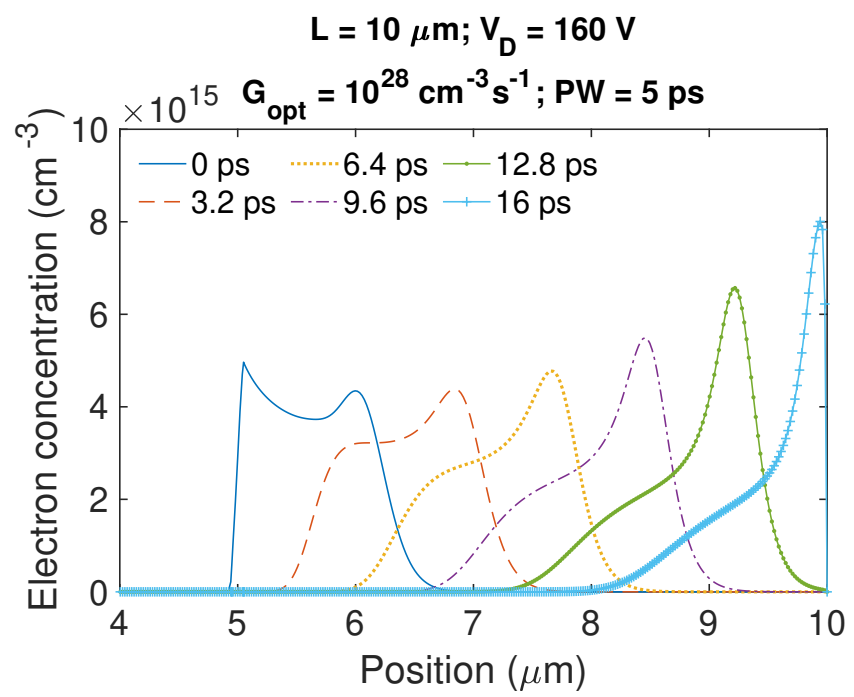

Fig. 7. Increase in the peak charge concentration and compression of pulse width as the charge cloud propagates along the length. The electric field across the device is $160 \mathrm{kV} / \mathrm{cm}$. The time that the laser is turned off is 0 ps.

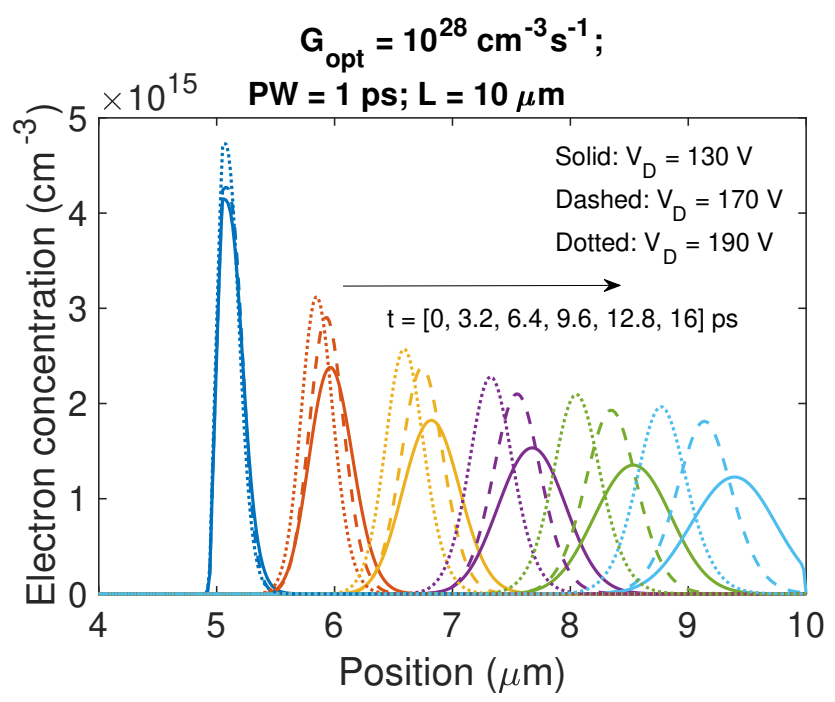

Fig. 8. Evolution of the charge cloud along the device length at various time instances after the laser is turned off at $\boldsymbol{t}=\mathbf{0}$.
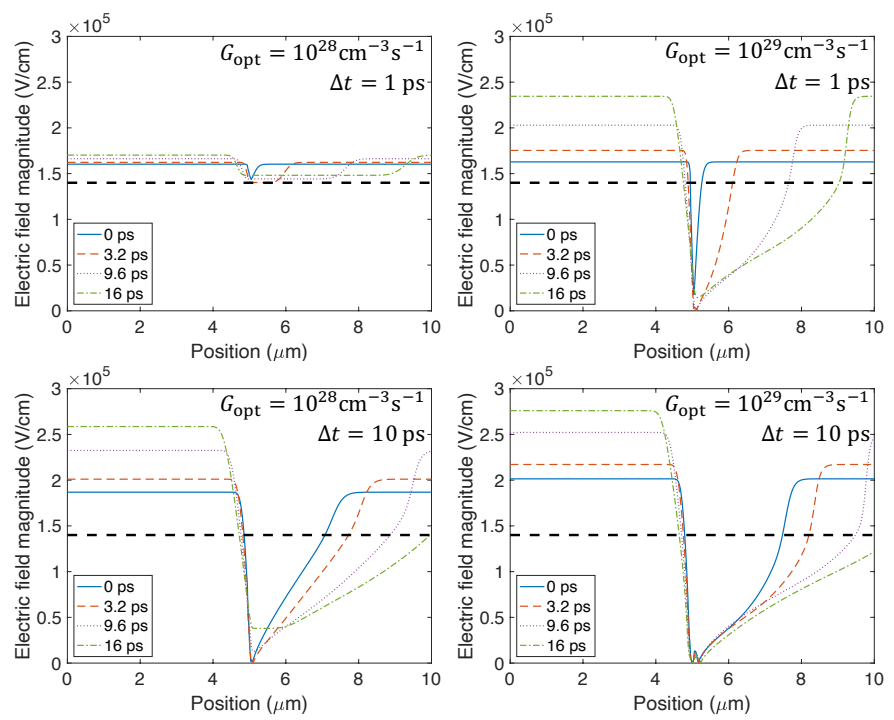

Fig. 9. Magnitude of the electrical field in the PCSS at different times. The length of the PCSS is $10 \mu \mathrm{m}$ and $V_{D}=160 \mathrm{~V}$. The bold dashed line indicates the electric field at the onset of NDM (i.e., $140 \mathrm{kV} / \mathrm{cm}$ ).

the density of optically excited carriers increases, which leads to strong electrostatic screening effects. At time $t=0$, the electric field in the middle of the channel is $1.44 \times 10^{5} \mathrm{~V} / \mathrm{cm}$ for $G_{\mathrm{opt}}=10^{28} \mathrm{~cm}^{-3} \mathrm{~s}^{-1}$ and $\Delta t=1 \mathrm{ps}$, but it drops to $10^{3} \mathrm{~V} / \mathrm{cm}$ for $\Delta t=10 \mathrm{ps}$. For $G_{\mathrm{opt}}=10^{29} \mathrm{~cm}^{-3} \mathrm{~s}^{-1}$ and $\Delta t=1 \mathrm{ps}$, the electric field in the middle of the channel is $1.9 \times 10^{4} \mathrm{~V} / \mathrm{cm}$ at $t=0$, while for $\Delta t=10 \mathrm{ps}$, the electric field in the middle of the channel is less than $10^{3} \mathrm{~V} / \mathrm{cm}$ at $t=0$. As the electron and hole charges are separated due to the applied bias, the electric field in the middle of the channel increases as $t$ increases. For large values of the product $G_{\text {opt }} \times \Delta t$, the electric field underneath the illumination region remains negligible indicating that a fraction of the charge carriers are stuck in the middle of the channel. Yet, the device maintains good turn-off characteristics as shown in Fig. 10. For $V_{D}=250 \mathrm{~V}$, the electron density under the illumination region starts dissipating at $\approx 300 \mathrm{ps}$. The electron current measured at the anode is fully dissipated for $t>180 \mathrm{ps}(320 \mathrm{ps})$ when $V_{D}=250 \mathrm{~V}(180 \mathrm{~V})$ in a $10-\mu \mathrm{m}$ PCSS with $G_{\text {opt }}=10^{29}$ 

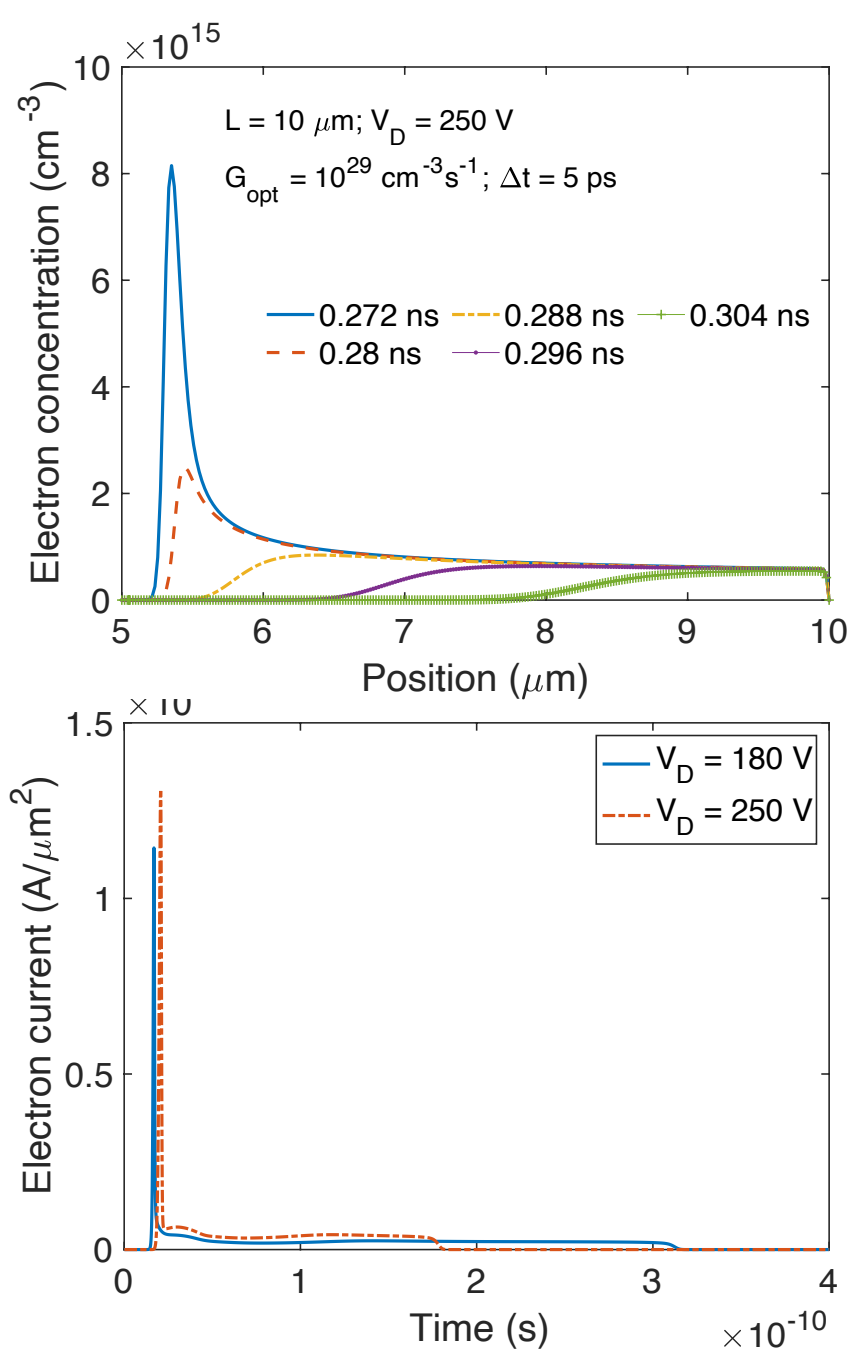

Fig. 10. (top) shows the electron concentration under the illumination region at various time points. (bottom) shows the measured electron current at anode for the same setup as in the top figure, but for different DC bias values.

$\mathrm{cm}^{-3} \mathrm{~s}^{-1}$ and laser pulse width of $1 \mathrm{ps}$.

Due to the pulse compression, the various FoMs of the GaN PCSS scale non-monotonically with electrical and optical bias fields. Figure 11 shows the non-monotonicity in bandwidth and maximum output power versus electric field for various $\mathrm{GaN}$ devices. Unlike in the case of GaAs, where significant electrostatic screening at high optical bias leads to signal distortion and performance reduction, in the case of $\mathrm{GaN}$, the performance can be pushed to much higher limits via engineering the electrical and optical bias applied to the device. As shown in Table II, GaN PCSS are suited for simultaneously achieving bandwidth $>500 \mathrm{GHz}$, output power $>800$ milli-watt with a power gain of $\approx 6000(=37.8 \mathrm{~dB})$. For all lengths, the peak output power occurs for $G_{\text {opt }}=10^{29}$ $\mathrm{cm}^{-3} \mathrm{~s}^{-1}$ and $\Delta t=1 \mathrm{ps}$, while the peak gain occurs for $G_{\mathrm{opt}}=10^{28} \mathrm{~cm}^{-3} \mathrm{~s}^{-1}$ and $\Delta t=10 \mathrm{ps}$. The maximum bandwidth is achieved for $G_{\mathrm{opt}}=10^{29} \mathrm{~cm}^{-3} \mathrm{~s}^{-1}$ and $\Delta t$ $=1$ ps for $L=10-\mu \mathrm{m}$ device, while for all other lengths, the maximum bandwidth occurs for $G_{\text {opt }}=10^{28} \mathrm{~cm}^{-3} \mathrm{~s}^{-1}$ and $\Delta t=5 \mathrm{ps}$. The DC electric field for achieving the best- case performance is shown in Fig. 12 for all device lengths. It can be seen from this figure that the DC electric field must be adjusted in the NDM regime, i.e., (190-260) $\mathrm{kV} / \mathrm{cm}$, to maximize the high-frequency performance of the PCSS. The performance metrics of the PCSS can be further improved via the engineering of the location of the laser spot along the device length as discussed in Sec. III.B.

TABLE II

COMPARISON OF THE HIGH-FREQUENCY AND HIGH-POWER METRICS OF GAN PCSS WITH VARYING LENGTH, I.E., THE SEPARATION BETWEEN THE CONTACTS. HERE, THE LASER IS INCIDENT IN THE MIDDLE OF THE CONTACTS. RESULTS ARE REPORTED FOR 100-NM LASER SPOT SIZE.

\begin{tabular}{c|c|c|c}
\hline Length $(\mu \mathrm{m})$ & Max. BW $(\mathrm{GHz})$ & $P_{\text {out }, \mathrm{m}}(\mathrm{mW})$ & $G_{\mathrm{pm}}(\mathrm{dB})$ \\
\hline 10 & 648.5 & 84.2 & 26.7 \\
25 & 578 & 204 & 31.6 \\
50 & 600 & 419 & 34.7 \\
100 & 609 & 836 & 37.8 \\
\hline
\end{tabular}

Figure 13 shows the impact of optical bias on the peak bandwidth of the PCSS of different lengths. For $\Delta t=1 \mathrm{ps,}$ the bandwidth increases as $G_{\text {opt }}$ increases and when the device length is scaled down. However, as the pulse width of the laser increases, the electrostatic screening effects also increase. This causes the bandwidth of the device to increase with an increase in the device length for $G_{\mathrm{opt}}>10^{28} \mathrm{~cm}^{-3} \mathrm{~s}^{-1}$. However, for $\Delta t=10 \mathrm{ps}$ and $G_{\mathrm{opt}}=10^{27} \mathrm{~cm}^{-3} \mathrm{~s}^{-1}$, the bandwidth decreases with an increase in the device length. Hence, it is the combination of $G_{\text {opt }}$ and $\Delta t$ which determines the net electrostatic screening effects present in the device, which lead to a non-Gaussian profile of the electron current at the anode.

\section{B. Off-center location of the laser source}

The location of the laser spot between the contacts is an important design choice to further enhance the performance of the GaN PCSS. For a $100-\mu \mathrm{m}$ GaN PCSS, the peak performance metrics (i.e., BW, $P_{\text {out,m }}$, and $G_{\mathrm{pm}}$ ) improve as the laser spot is moved closer to the anode as shown in Fig. 14. To observe the peak performance, the DC electric field across the device must be increased to overcome the electrostatic screening effects associated with large carrier densities generated closer to the anode. Our comprehensive results listed in Table III show that a $100-\mu \mathrm{m}$ GaN PCSS can deliver $P_{\text {out }, \mathrm{m}} \sim 1500 \mathrm{~mW}$ with $G_{\mathrm{pm}}>40 \mathrm{~dB}$ while operating at near- $\mathrm{THz}$ frequency if the laser is incident at $12.5 \mu \mathrm{m}$ distance from the anode. The optical bias required to achieve the best-in class performance is $G_{\mathrm{opt}}=10^{28} \mathrm{~cm}^{-3} \mathrm{~s}^{-1}$ and $\Delta t=10 \mathrm{ps}$, while the electric field requirement changes as $\Delta_{s}$ changes. However, for all cases of $\Delta_{s}$ examined in our work, the DC electric field must be set greater than $190 \mathrm{kV} / \mathrm{cm}$ to benefit from the NDM effect in GaN.

\section{Conclusions}

In this paper, we presented a novel photoconductive semiconductor switch (PCSS) based on GaN to generate highvoltage signals at near-terahertz frequencies $(>500 \mathrm{GHz})$ in 

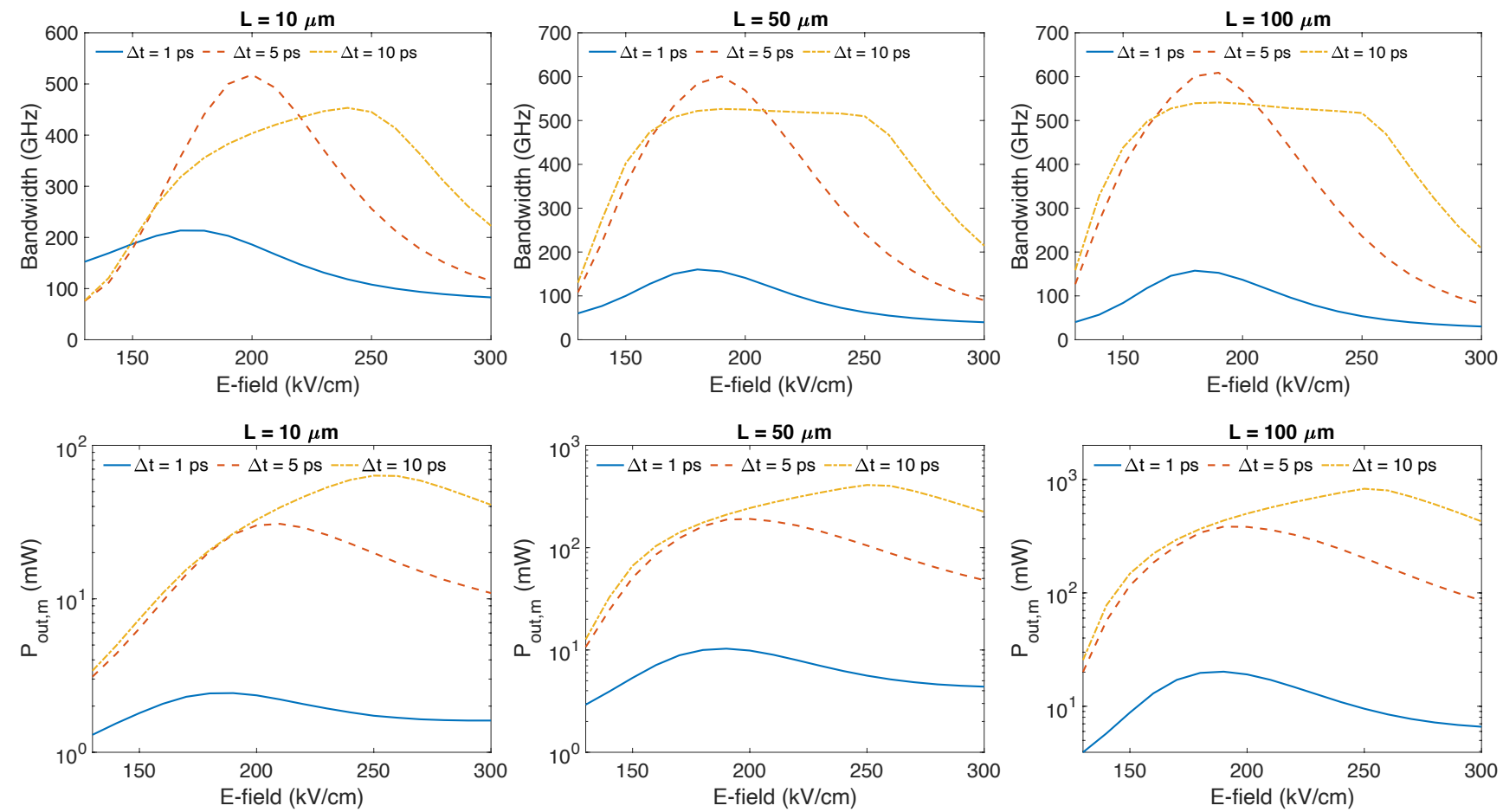

Fig. 11. (top panel) Bandwidth versus DC electric field and (bottom panel) maximum output power versus DC electric field of GaN PCSS at different laser pulse widths and device lengths. The optical generation rate is fixed at $10^{28} \mathrm{~cm}^{-3} \mathrm{~s}^{-1} . \Delta t$ denotes the pulse width of the laser.

TABLE III

BY ENGINEERING THE LOCATION OF THE LASER SPOT BETWEEN THE CONTACTS, THE HIGH-FREQUENCY AND HIGH-POWER PERFORMANCE OF THE DEVICE CAN BE BOOSTED. DEVICE LENGTH IS $100 \mu \mathrm{M}$ AND THE SIZE OF THE LASER SPOT IS $100 \mathrm{NM}$. THE BOTTOM ROW FOR EACH $\boldsymbol{\Delta}_{s}$ VALUE SHOWS THE DC ELECTRIC FIELD REQUIRED TO ACHIEVE THE BEST-CASE PERFORMANCE. THE COLORED CELLS INDICATE THE BEST-IN CLASS PERFORMANCE OF THE GAN PCSS.

\begin{tabular}{|c|c|c|c|c|c|c|}
\hline \multirow{2}{*}{$\Delta_{s}(\mu \mathrm{m})$} & \multicolumn{3}{|c|}{$G_{\mathrm{opt}}=10^{28} \mathrm{~cm}^{-3} \mathrm{~s}^{-1}$ and $\Delta t=5 \mathrm{ps}$} & \multicolumn{3}{|c|}{$G_{\mathrm{opt}}=10^{28} \mathrm{~cm}^{-3} \mathrm{~s}^{-1}$ and $\Delta t=10 \mathrm{ps}$} \\
\hline & BW (GHz) & $P_{\text {out }, \mathrm{m}}$ (Watts) & $G_{\mathrm{pm}}(\mathrm{dB})$ & BW (GHz) & $P_{\text {out }, \mathrm{m}}$ (Watts) & $G_{\mathrm{pm}}(\mathrm{dB})$ \\
\hline & 609 & 0 . & 34.5 & 54 & 0.829 & 37.8 \\
\hline & $190 \mathrm{kV} / \mathrm{cm}$ & $190 \mathrm{kV} / \mathrm{cm}$ & $190 \mathrm{kV} / \mathrm{cm}$ & $190 \mathrm{kV} / \mathrm{cm}$ & $250 \overline{\mathrm{kV}} / \mathrm{cm}$ & $250 \overline{\mathrm{kV} / \mathrm{cm}}$ \\
\hline \multirow{2}{*}{7.5} & 624 & 0 . & 34.8 & 663 & 1. & 39 \\
\hline & $200 \mathrm{kV} / \mathrm{cm}$ & $210 \mathrm{kV} / \mathrm{cm}$ & $210 \mathrm{kV} / \mathrm{cm}$ & $230 \mathrm{kV} / \mathrm{cm}$ & $270 \mathrm{kV} / \mathrm{cm}$ & $270 \mathrm{kV} / \mathrm{cm}$ \\
\hline & 624 & 0.45 & 35.2 & 755 & 1.33 & 40.0 \\
\hline & $210 \mathrm{kV} / \overline{\mathrm{cm}}$ & $230 \mathrm{kV} / \mathrm{cm}$ & $230 \mathrm{kV} / \mathrm{cm}$ & $\overline{270} \overline{\mathrm{kV}} \overline{\mathrm{cm}} \overline{\mathrm{m}}$ & $\overline{2} 9 \overline{\mathrm{kV}} / \overline{\mathrm{cm}}$ & $\overline{2} 9 \overline{\mathrm{kV} / \mathrm{cm}}$ \\
\hline \multirow{2}{*}{12.5} & 626 & 0.49 & 35.5 & 798 & 1.51 & 40.4 \\
\hline & $230 \mathrm{kV} / \mathrm{cm}$ & $240 \mathrm{kV} / \mathrm{cm}$ & $240 \mathrm{kV} / \mathrm{cm}$ & $290 \mathrm{kV} / \mathrm{cm}$ & $320 \mathrm{kV} / \mathrm{cm}$ & $320 \mathrm{kV} / \mathrm{cm}$ \\
\hline
\end{tabular}

a compact form-factor. The GaN PCSS utilizes the unique negative differential mobility (NDM) effect in the conduction band of GaN to simultaneously compress the pulse width of the output current and increase its magnitude, thus providing output power on the order of a watt, power gain $>40 \mathrm{~dB}$, while operating with $>500 \mathrm{GHz}$ bandwidth at room temperature. These performance metrics clearly exceed those achieved with GaN and InP RF high-electron mobility transistors. Compared to other high-power and high-frequency technologies, including traveling wave tubes, extended interaction kylstrons, and backwards wave oscillators, GaN PCSS can be readily miniaturized to area under few $\mathrm{cm}^{2}$. Depending on the device geometry, there exists an optimal combination of electrical and optical bias conditions that maximizes the device bandwidth, output power, and power gain. When the laser is incident in the middle of the device, the device performance is maximized for DC electric fields in the NDM regime (i.e., 190 to $260 \mathrm{kV} / \mathrm{cm}$ ) with optical generation rate of $10^{28} \mathrm{~cm}^{-3} \mathrm{~s}^{-1}$ to $10^{29} \mathrm{~cm}^{-3} \mathrm{~s}^{-1}$ and laser pulse width in the (1-5) ps range. When the laser source is incident closer to the anode, the DC electric fields must be increased to achieve the upper bound of performance. For example, a $100-\mu \mathrm{m}$ device with laser incident at a distance of $12.5 \mu \mathrm{m}$ from the anode can operate at $\approx 800 \mathrm{GHz}$, while delivering output power of 1.5 Watts and power gain of $40 \mathrm{~dB}$. However, these metrics are achieved only when the DC electric field in the device is pushed to (290-320) $\mathrm{kV} / \mathrm{cm}$. Our future work will understand the impact of heating due to the highly intense laser source on the propagation characteristics of the 


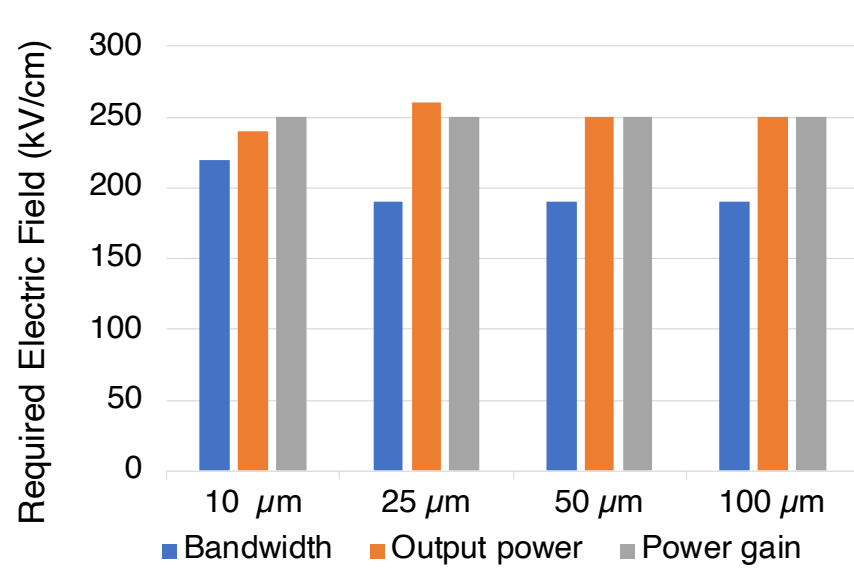

Fig. 12. Electric field required to maximize bandwidth, output power, and power gain for GaN PCSS with different lengths. The optical bias for the best-case performance depends on the device length as discussed in the text.

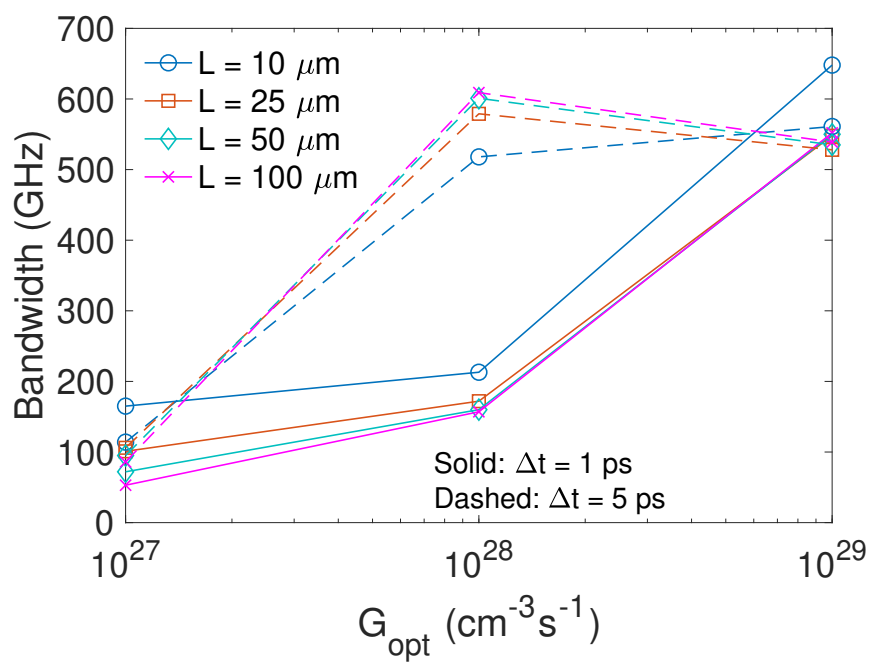

Fig. 13. Scaling of the peak bandwidth of the device with optical bias and device length. The electric field is different for each device length and optical bias point.

charge cloud, as well as understanding the device operation by considering a coupled electrical-optical simulation framework that accurately interprets the two-dimensional effects.

\section{REFERENCES}

[1] R. Baldemair, E. Dahlman, G. Fodor, G. Mildh, S. Parkvall, Y. Selen, $\mathrm{H}$. Tullberg, and K. Balachandran, "Evolving wireless communications: Addressing the challenges and expectations of the future," IEEE Vehicular Technology Magazine, vol. 8, no. 1, pp. 24-30, 2013.

[2] A. Hirata and M. Yaita, "Ultrafast terahertz wireless communications technologies," IEEE Transactions on Terahertz Science and Technology, vol. 5, no. 6, pp. 1128-1132, 2015.

[3] H. Elayan, O. Amin, R. M. Shubair, and M.-S. Alouini, "Terahertz communication: The opportunities of wireless technology beyond 5G," in 2018 International Conference on Advanced Communication Technologies and Networking (CommNet). IEEE, 2018, pp. 1-5.

[4] H. Hamada, T. Fujimura, I. Abdo, K. Okada, H.-J. Song, H. Sugiyama, H. Matsuzaki, and H. Nosaka, "300-GHz 100-Gb/s InP-HEMT wireless transceiver using a 300-GHz fundamental mixer," in 2018 IEEE/MTT-S International Microwave Symposium-IMS. IEEE, 2018, pp. 1480-1483.
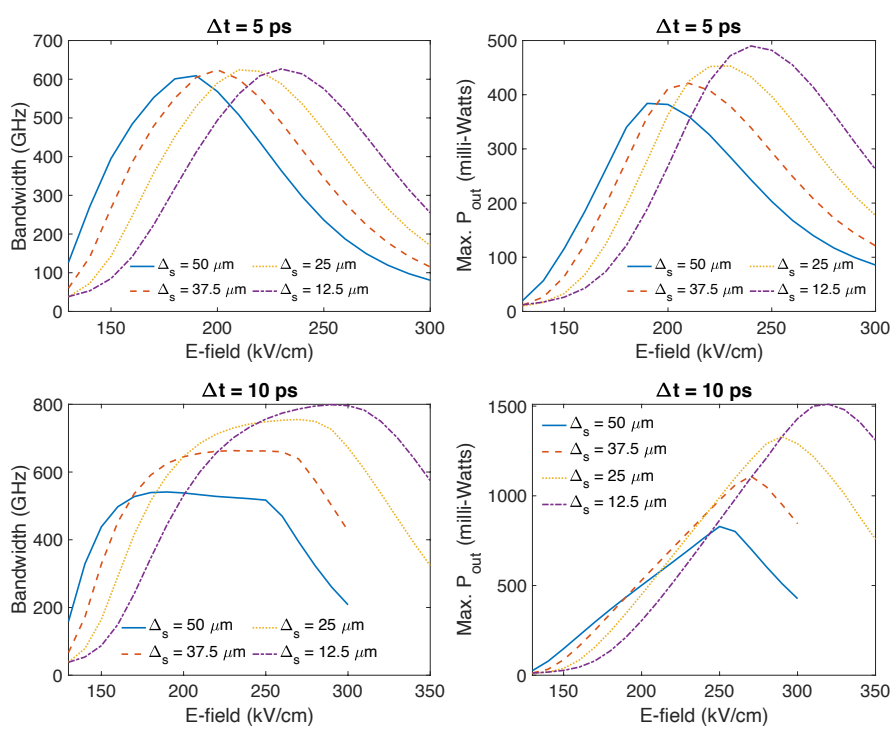

Fig. 14. Impact of the location of the laser spot, measured from the anode $\left(\Delta_{s}\right)$, on the performance metrics of the GaN PCSS of length $100 \mu \mathrm{m}$. Results are reported for $G_{\mathrm{opt}}=10^{28} \mathrm{~cm}^{-3} \mathrm{~s}^{-1}$.

[5] H. Hamada, T. Tsutsumi, H. Matsuzaki, T. Fujimura, I. Abdo, A. Shirane, K. Okada, G. Itami, H.-J. Song, H. Sugiyama et al., "300GHz-Band 120-Gb/s Wireless Front-End Based on InP-HEMT PAs and Mixers," IEEE Journal of Solid-State Circuits, vol. 55, no. 9, pp. 2316$2335,2020$.

[6] A. Khalid, N. Pilgrim, G. Dunn, M. Holland, C. Stanley, I. Thayne, and D. Cumming, "A planar Gunn diode operating above $100 \mathrm{GHz}$," IEEE Electron Device Letters, vol. 28, no. 10, pp. 849-851, 2007.

[7] A. Khalid, G. Dunn, R. Macpherson, S. Thoms, D. Macintyre, C. Li, M. Steer, V. Papageorgiou, I. Thayne, M. Kuball et al., "Terahertz oscillations in an $\mathrm{In}_{0.53} \mathrm{Ga}_{0.47}$ As submicron planar Gunn diode," Journal of Applied Physics, vol. 115, no. 11, p. 114502, 2014.

[8] C. Sirtori, S. Barbieri, and R. Colombelli, "Wave engineering with $\mathrm{THz}$ quantum cascade lasers," Nature Photonics, vol. 7, no. 9, pp. 691-701, 2013.

[9] B. Hinkov, A. Hugi, M. Beck, and J. Faist, "Rf-modulation of midinfrared distributed feedback quantum cascade lasers," Optics Express, vol. 24, no. 4, pp. 3294-3312, 2016.

[10] O. Scholder, "Fabrication, simulation and characterization of tunable plasmonic nano antennas," Ph.D. dissertation, ETH Zurich, 2014.

[11] R. Adato, A. A. Yanik, and H. Altug, "On chip plasmonic monopole nano-antennas and circuits," Nano letters, vol. 11, no. 12, pp. 5219$5226,2011$.

[12] D. Gamzina, X. Li, C. Hurd, Y. Tang, X. Huang, Y. Zheng, L. Himes, M. Gonzalez, H. Li, P. Pan et al., "Backward wave oscillator for high power generation at THz frequencies," in Terahertz Emitters, Receivers, and Applications VIII, vol. 10383. International Society for Optics and Photonics, 2017, p. 1038303.

[13] P. Howson, R. Miller, and D. Ryder, "Photoconductive switch design and applications," in IEEE International Symposium on Electrical Insulation. IEEE, 1990, pp. 461-464.

[14] R. Quay, Gallium nitride electronics. Springer Science \& Business Media, 2008, vol. 96.

[15] B. R. Pamplin, "Negative differential conductivity effects in semiconductors," Contemporary Physics, vol. 11, no. 1, pp. 1-19, 1970.

[16] Z. Huang, R. Goldberg, J. Chen, Y. Zheng, D. B. Mott, and P. Shu, "Direct observation of transferred-electron effect in GaN," Applied physics letters, vol. 67, no. 19, pp. 2825-2826, 1995.

[17] C. Sevik and C. Bulutay, "Gunn oscillations in GaN channels," Semiconductor science and technology, vol. 19, no. 4, p. S188, 2004.

[18] E. Alekseev and D. Pavlidis, "GaN Gunn diodes for $\mathrm{THz}$ signal generation," in 2000 IEEE MTT-S International Microwave Symposium Digest (Cat. No. 00CH37017), vol. 3. IEEE, 2000, pp. 1905-1908.

[19] S. Bloom, G. Harbeke, E. Meier, and I. B. Ortenburger, "Band Structure and Reflectivity of GaN," physica status solidi (b), vol. 66, no. 1, pp. 161-168, 1974. [Online]. Available: https://onlinelibrary.wiley.com/doi/abs/10.1002/pssb.2220660117 
[20] P. A. Markowich, C. A. Ringhofer, and C. Schmeiser, Semiconductor equations. Springer Science \& Business Media, 2012.

[21] W. Shockley and W. T. Read, "Statistics of the recombinations of holes and electrons," Phys. Rev., vol. 87, pp. 835-842, Sep 1952. [Online]. Available: https://link.aps.org/doi/10.1103/PhysRev.87.835

[22] T. Goudon, V. Miljanović, and C. Schmeiser, "On the ShockleyRead-Hall model: generation-recombination in semiconductors," SIAM Journal on Applied Mathematics, vol. 67, no. 4, pp. 1183-1201, 2007.

[23] J. Meyer, R. Liu, R. Schaller, H.-P. Lee, and C. Bayram, "Systematic study of shockley-read-hall and radiative recombination in gan on al 2 o 3, freestanding gan, and gan on si," Journal of Physics: Photonics, 2020.

[24] J. Mickevičius, M. Shur, R. Q. Fareed, J. Zhang, R. Gaska, and G. Tamulaitis, "Time-resolved experimental study of carrier lifetime in gan epilayers," Applied Physics Letters, vol. 87, no. 24, p. 241918, 2005.

[25] S. Juršènas, N. Kurilčik, G. Kurilčik, S. Miasojedovas, A. Žukauskas, T. Suski, P. Perlin, M. Leszczynski, P. Prystawko, and I. Grzegory, "Optical gain in homoepitaxial gan," Applied physics letters, vol. 85, no. 6, pp. 952-954, 2004.

[26] S. Chichibu, H. Marchand, M. Minsky, S. Keller, P. Fini, J. Ibbetson, S. Fleischer, J. Speck, J. Bowers, E. Hu et al., "Emission mechanisms of bulk gan and ingan quantum wells prepared by lateral epitaxial overgrowth," Applied physics letters, vol. 74, no. 10, pp. 1460-1462, 1999.

[27] J. Im, A. Moritz, F. Steuber, V. Härle, F. Scholz, and A. Hangleiter "Radiative carrier lifetime, momentum matrix element, and hole effective mass in GaN," Applied physics letters, vol. 70, no. 5, pp. 631-633, 1997.

[28] A. Dmitriev and A. Oruzheinikov, "The rate of radiative recombination in the nitride semiconductors and alloys," Journal of applied physics, vol. 86, no. 6, pp. 3241-3246, 1999.

[29] D. R. Naylor, A. Dyson, and B. K. Ridley, "Steady-state and transient electron transport in bulk GaN employing an analytic bandstructure," Solid state communications, vol. 152, no. 6, pp. 549-551, 2012.

[30] B. E. Foutz, S. K. O'Leary, M. S. Shur, and L. F. Eastman, "Transient electron transport in wurtzite GaN, InN, and AlN," Journal of Applied Physics, vol. 85, no. 11, pp. 7727-7734, 1999.

[31] J. Barker, D. Ferry, D. Koleske, and R. Shul, "Bulk GaN and Al$\mathrm{GaN} / \mathrm{GaN}$ heterostructure drift velocity measurements and comparison to theoretical models," Journal of applied physics, vol. 97, no. 6, p. 063705,2005

[32] M. Farahmand, C. Garetto, E. Bellotti, K. F. Brennan, M. Goano, E. Ghillino, G. Ghione, J. D. Albrecht, and P. P. Ruden, "Monte Carlo simulation of electron transport in the III-nitride wurtzite phase materials system: binaries and ternaries," IEEE Transactions on electron devices, vol. 48, no. 3, pp. 535-542, 2001.

[33] V. M. Polyakov and F. Schwierz, "Influence of electron mobility modeling on DC IV characteristics of WZ-GaN MESFET," IEEE Transactions on Electron Devices, vol. 48, no. 3, pp. 512-516, 2001.

[34] F. Bertazzi, M. Moresco, and E. Bellotti, "Theory of high field carrier transport and impact ionization in wurtzite GaN. Part I: a full band Monte Carlo model," Journal of Applied Physics, vol. 106, no. 6, p. 063718, 2009

[35] A. Reklaitis and L. Reggiani, "Monte carlo study of hot-carrier transport in bulk wurtzite GaN and modeling of a near-terahertz impact avalanche transit time diode," Journal of applied physics, vol. 95, no. 12, pp. 79257935, 2004.

[36] C. G. Rodrigues, Á. R. Vasconcellos, and R. Luzzi, "Nonlinear hole transport and nonequilibrium thermodynamics in group III-nitrides under the influence of electric fields," Journal of Applied Physics, vol. 102, no. 7, p. 073714, 2007

[37] S. Chen and G. Wang, "High-field properties of carrier transport in bulk wurtzite GaN: A Monte Carlo perspective," Journal of Applied Physics, vol. 103, no. 2, p. 023703, 2008.

[38] D. Ji, B. Ercan, and S. Chowdhury, "Experimental determination of velocity-field characteristic of holes in GaN," IEEE Electron Device Letters, vol. 41, no. 1, pp. 23-25, 2019.

Shaloo Rakheja is currently an Assistant Professor of Electrical and Computer Engineering with the University of Illinois at UrbanaChampaign, Urbana, IL, where she is involved in physical modeling and numerical simulation of nanoelectronic and nanomagnetic devices. She was a Postdoctoral Research Associate with Microsystems Technology
Laboratories, Massachusetts Institute of Technology, Cambridge, MA, USA, from 2013 to 2014. She received her Ph.D. and M.S. in Electrical and Computer Engineering from the Georgia Institute of Technology in 2012 and 2009 , respectively.

Kexin Li is currently working toward a $\mathrm{PhD}$ degree in Electrical and Computer Engineering from the University of Illinois at UrbanaChampaign, Urbana, IL. Her research focus is on the modeling and simulation of ultrafast optoelectronic devices for RF applications.

Karen M. Dowling received her PhD degree in Electrical Engineering from Stanford University in 2019. She is currently a Postdoctoral Research Staff Member at the Lawrence Livermore National Laboratory. Her research focus is on widebandgap materials for creating devices in high power, high speed applications.

Lars F. Voss is a materials scientist and the Group Leader for MEMS, Electronics, and Photonics Technologies at LLNL. He specializes in semiconductor materials, devices, and microfabrication. He previously completed a postdoctoral appointment at LLNL and his $\mathrm{PhD}$ and MS in Materials Science from the University of Florida.

Adam M. Conway Received his B.S. in Electrical Engineering from Northwestern University (2000) and M.S and Ph.D. from University of California, San Diego (2006) under Prof. Peter Asbeck. He joined Lawrence Livermore National Laboratory in 2007. His research interests include semiconductor radiation detectors, wideband gap semiconductors and high power optoelectronic devices. 